\title{
Enhanced Gastric Emptying Scintigraphy to Assess Fundic Accommodation Using Intragastric Meal Distribution and Antral Contractility
}

\author{
Perry Orthey, Simin Dadparvar, Henry P. Parkman, and Alan H. Maurer \\ Section of Gastroenterology, Department of Medicine, and Section of Nuclear Medicine, Department of Radiology, Temple University \\ School of Medicine, Philadelphia, Pennsylvania
}

See an invited perspective on this article on page 137.

Gastric emptying scintigraphy (GES) as now commonly performed measures only total gastric emptying. Intragastric meal distribution (IMD) immediately after meal ingestion ( $t=0 \mathrm{~min}$ ) $\left(\mathrm{IMD}^{\circ}\right)$ can assess fundic accommodation, and dynamic antral contraction scintigraphy (DACS) can assess antral motility. Our goals were to incorporate IMD and DACS into GES, compare $\mathrm{IMD}^{0}$ using gastric division into anatomic proximal and distal halves versus more physiologic separation of the antrum from the proximal stomach using DACS, and establish reference values. Methods: Healthy subjects $(n=20)$ underwent GES using a solid-liquid meal. DACS (1 frame/3 s) was performed for $20 \mathrm{~min}$ after each static imaging time. $\mathrm{IMD}^{0}$ was measured using both semiautomated software to divide the gastric long axis into anatomic halves and Fourier analysis to identify antral pixels with phasic contractions. Results: Using halving of the stomach, IMD ${ }^{0}$ averaged $0.75 \pm 0.15(\mathrm{SD})$. Using phasic contractions to define the antrum, mean $\mathrm{IMD}^{0}$ was $0.85 \pm 0.14(P=$ $0.004)$. Sustained antral contractions started at a mean of 11.24 \pm 12.98 min after meal ingestion and originated in the gastric midbody with a starting location at $40.5 \% \pm 10.8 \%$ from the distal to the proximal stomach along its long axis. Antral frequency and ejection fraction peaked 30 min after meal ingestion at $3.30 \pm 0.71$ contractions per minute and an ejection fraction of $30.3 \% \pm 13.69 \%$, when mean antral filling peaked at $36.7 \% \pm$ $14 \%$. Maximum antral contraction speed was $3.54 \pm 0.90 \mathrm{~mm} / \mathrm{s}$ at $60 \mathrm{~min}$ after meal ingestion. Gastric retention was $39.8 \% \pm$ $12.8 \%$ at $2 \mathrm{~h}$ and $5.8 \% \pm 6.0 \%$ at $4 \mathrm{~h}$. Conclusion: Addition of DACS to GES permits physiologic characterization of both fundic accommodation and antral contractility to supplement routine GES.

Key Words: gastric emptying; fundic accommodation; antral contractility; gastroparesis

J Nucl Med Technol 2019; 47:138-143

DOI: 10.2967/jnmt.118.215566

\footnotetext{
Received Jun. 1, 2018; revision accepted Aug. 17, 2018.

For correspondence or reprints contact: Alan H. Maurer, Gastroenterology Section; Parkinson Pavilion, 8th Floor, Temple University School of Medicine, 3500 N. Broad St, , Philadelphia, PA 19140.

E-mail: amaurer@temple.edu

Published online Aug. 23, 2018.

COPYRIGHT (c) 2019 by the Society of Nuclear Medicine and Molecular Imaging.
}

G astric emptying scintigraphy (GES) as currently performed is used to assess total gastric emptying. In standard GES, anterior and posterior static images are taken every hour after meal ingestion over $4 \mathrm{~h}(1)$. Processing of the static images requires decay correction, geometric mean correction of the total gastric counts in the anterior and posterior images to compensate for depth correction, and then normalization of total gastric counts to $100 \%$ immediately after ingestion ( $\mathrm{t}=0 \mathrm{~min}$ ) to calculate the percentage of gastric counts remaining in the entire stomach at each time point (2). GES as now typically performed, however, for many symptomatic patients results in normal total gastric emptying (3). This problem has resulted in increasing interest in obtaining more information on the role of impaired fundic accommodation and antral dysmotility to explain symptoms and help direct targeted therapy (4).

Measurement of total gastric emptying does not adequately reflect the separate functions of the proximal and distal stomach. The fundus, functionally the proximal portion of the stomach, normally undergoes receptive relaxation and accommodation and then generates sustained pressure to move solids into the antrum. In response, the antrum then performs rhythmic contractions to break down the solid food particles to permit emptying of the solids through the pylorus.

Proximal gastric function and fundic accommodation can be assessed with routine GES imaging. Intragastric meal distribution (IMD) is defined as a measurement of how much food is in the proximal stomach compared with the food in the whole stomach. To use IMD as a measure of fundic accommodation, the IMD immediately after meal ingestion, or $\mathrm{IMD}^{0}$, has previously been calculated by anatomic division of the stomach into halves $(5,6)$ or thirds (7).

Measurement of antral contractions can also be obtained as a part of GES with the addition of continuous dynamic imaging. Dynamic antral contraction scintigraphy (DACS) has been previously described and uses continuous imaging typically at 1 image per $3 \mathrm{~s}(8-10)$. Using an antral region 
of interest (ROI) and Fourier analysis, this can yield information on the frequency of antral contractions and assessment of the strength of antral contractions by measuring an antral ejection fraction. Although not previously described, an additional advantage of DACS is its ability to use the phasic and rhythmic contractions of the antrum to functionally separate the antrum from the noncontractile proximal stomach and thus permit a more physiologic measurement of $\mathrm{IMD}^{0}$ than is possible with simple anatomic division of the stomach.

The ability to measure abnormal fundic accommodation and abnormal antral motility using GES is important because they may potentially explain dyspeptic symptoms that are not explained by GES as currently performed $(11,12)$. Studies using the gastric barostat suggested that impaired fundic accommodation is associated with early satiety and weight loss (13). Although the barostat study is considered the gold standard for assessing fundic accommodation, it is invasive and not widely available. In addition, the barostat balloon itself can alter gastric physiology (14). Several alternate methods have been developed to measure fundic accommodation, such as SPECT and MRI, but these are not in widespread use or use technology not widely available (14).

The primary aim of this study was to develop an acquisition protocol and software for GES that would permit measurement of IMD and DACS frequency and amplitude measurements in routine, clinical GES. Secondary aims were to establish reference values for IMD and DACS and to compare measurement of IMD ${ }^{0}$ using DACS to physiologically define the antrum versus simple anatomic division of the stomach.

\section{MATERIALS AND METHODS}

After obtaining institutional review board approval for this study of healthy volunteers, we recruited subjects to undergo GES with DACS. These subjects gave written informed consent and were questioned to ensure they had no prior history of gastrointestinal disease or dysfunction, had no prior gastrointestinal surgery, and were not taking medications that might affect gastrointestinal function.

\section{GES}

Subjects came to the nuclear medicine department in the morning after fasting overnight. GES was measured using the 4-h, liquid-egg-white protocol described initially by Tougas et al. (15) and currently recommended by the consensus report of the Society of Nuclear Medicine and Molecular Imaging and the American Neurogastroenterology and Motility Society (1). The

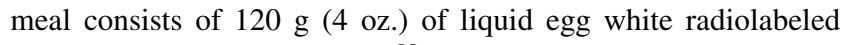
with $74.0 \mathrm{MBq}(2.0 \mathrm{mCi})$ of ${ }^{99 \mathrm{~m}} \mathrm{Tc}$-sulfur colloid served with 2 pieces of white bread and jelly. In addition, patients were given $120 \mathrm{~mL}$ of water immediately after ingestion of the solid portion of the meal. The current Society of Nuclear Medicine and Molecular Imaging procedure guideline for GES (version 3.0) recom-

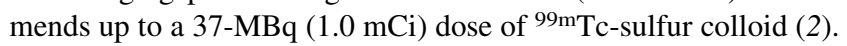
The higher dose used for this study was to ensure adequate count rates for analysis of the DACS based on prior experience (16).
After meal ingestion, static imaging using a $128 \times 128$ matrix was performed at $0,0.5,1,2,3$, and $4 \mathrm{~h}$, with the subject upright in the anterior and then the posterior position each for $30 \mathrm{~s}$. Subjects then underwent DACS imaging as previously described (16). This consisted of continuous anterior imaging ( 1 frame/3 s) for $20 \mathrm{~min}$ beginning immediately after each of the anterior and posterior static image sets. For the DACS imaging, each subject was allowed to choose whether an upright standing or seated position was most comfortable and then instructed to remain as still as possible in that position for the $20 \mathrm{~min}$ of DACS imaging. In between the imaging times, the subjects sat in the nuclear medicine waiting area.

Before processing of the DACS images, the continuous dynamic images were reviewed and motion-corrected to eliminate any motion artifacts using GE Xeleris (GE Healthcare) motion correction software.

All GES images were analyzed using image processing software developed for this project. The software was developed using Matlab (MathWorks). A more detailed and technical description of the software is provided in the supplemental appendix (supplemental materials are available at http://jnmt.snmjournals.org). The overall image processing included measurement of the following:

Total Gastric Emptying. Static image processing included standard decay correction, geometric mean attenuation correction, and calculation of the percentage remaining at each time point (2).

IMD Based on Partitioning of Proximal and Distal Stomach by Halves. IMD was quantitatively measured with Matlab semiautomated software, as previously described (6). This software first defines an ROI for the total stomach using a summation of all coregistered anterior static gastric images (Fig. 1A). The software then divides the stomach into proximal and distal halves along the longitudinal axis of the stomach. The longitudinal axis is found by taking the midpoints between the ROI that defines the inner curvature and outer curvature of the stomach, starting from the most distal stomach boundary and moving toward its most proximal point. A third-degree polynomial is used to smooth the midpoints and to define the longitudinal axis. The midpoint $(50 \%$ of the distance along the longitudinal axis) is used as the reference point to divide the distal half from the proximal half of the stomach

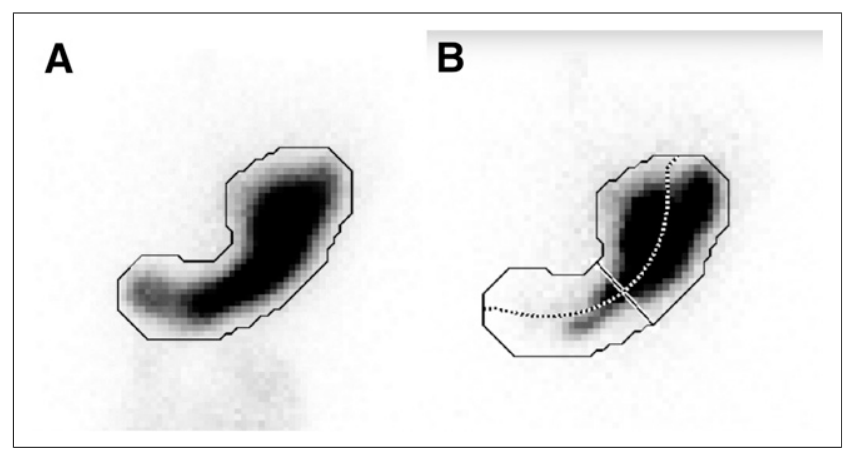

FIGURE 1. Anatomic division of stomach into proximal and distal halves by semiautomated software. (A) Demonstration of how software automatically contours outer border for totalstomach ROI (solid line) from summed set of all static anterior images. (B) Application of total-stomach ROI to each static image. In this case, image is anterior image acquired immediately after meal ingestion. By finding midpoint between opposing points of total-stomach ROI, longitudinal axis through stomach is generated (dotted line). Line perpendicular to longitudinal axis is then defined at point dividing longitudinal axis into equal halves to separate upper and lower segments of stomach. 
(Fig. 1B). These regions are separated by a line that goes through the reference point and is perpendicular to the longitudinal axis. Measurement of $\mathrm{IMD}^{0}$ using the proximal half of the stomach is defined as the ratio of counts in the proximal half of the stomach to the total gastric counts, immediately after meal ingestion.

IMD Based on Partitioning of Proximal Stomach from Contracting Antrum (Distal Stomach) by DACS Fourier Analysis. We used the method of fast Fourier transformation to automatically differentiate the noncontracting proximal stomach from the distal contracting antrum. Fast Fourier transformation of the time-activity curve of counts from a vertical ROI in the midportion of the antrum was performed as previously described (16). Fourier analysis reveals which pixels demonstrate regular, rhythmic contractions and a dominant frequency. The gastric antrum was then defined as that portion of the stomach containing these contracting pixels. The remaining area of the stomach was specified as the noncontracting proximal stomach (Fig. 2).

Additional values derived from the fast Fourier transformation DACS analysis included time of onset of sustained antral contractions after meal ingestion (Fig. 3), the location of the site of origination of antral contractions referenced to the long axis (Fig. 2C), the dominant frequency of contractions, the antral ejection fraction; and the speed of propagation of the antral contractions. The antral ejection fraction is a measure of the amplitude of antral contractions, defined by the percentage change in measured dynamic time-activity curve of the mid antral ROI created by the propagating contracting waves (supplemental appendix).

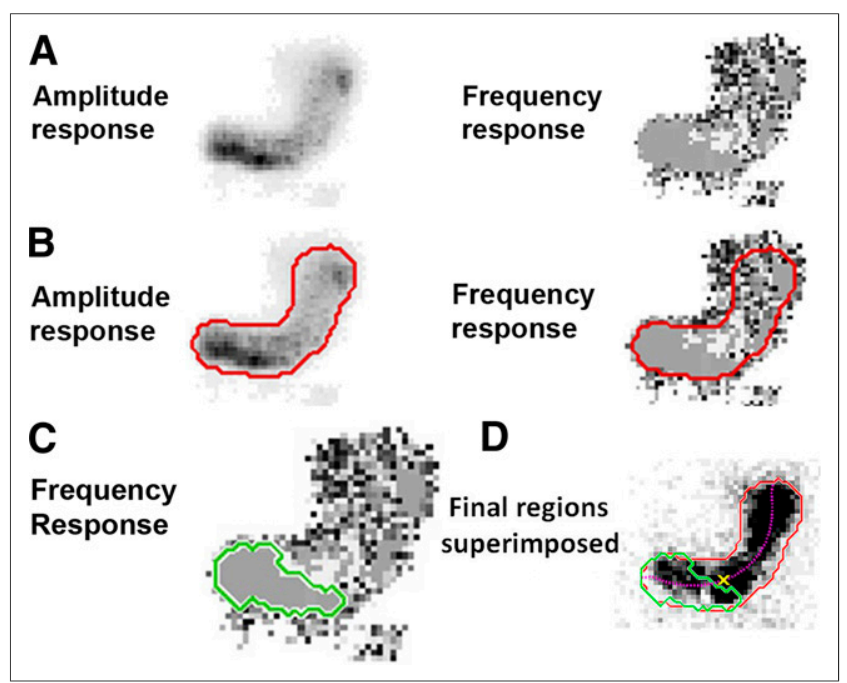

FIGURE 2. Fourier frequency and amplitude analysis used to segment antrum (distal stomach) from proximal stomach based on antral contractions. (A) Fast Fourier transformation of all pixels in DACS image set: amplitude and frequency response. (B) Red border on amplitude response indicating threshold of high-amplitude pixels. Same border is applied to frequency response. (C) Region of dominant frequency. Green border indicates contiguous region of pixels that have same frequency (gray color). (D) Starting location of antral contractions. Antrumdefined ROI (green border) using region of dominant frequency from $C$ and red border from $B$ is applied to one image of gastric emptying study. Purple dotted line is longitudinal axis as shown in Figure 1B. Yellow $X$ is intersection of longitudinal axis, and convex boundary around green border is starting location of antral contractions.

\section{Statistical Analysis}

The results were entered into a Microsoft Excel database. Reported results are expressed as mean \pm SD. The Student $t$ test was used to test differences between values. Spearman correlation was used to correlate different parameters using the descriptors very weak (0.00-0.19), weak (0.20-0.39), moderate $(0.40-0.59)$, strong (0.60-0.79), and very strong (0.80-1.0) (17).

\section{RESULTS}

\section{Study Subjects}

Twenty-one subjects were studied. One subject was excluded from the final analysis because of excessive motion artifacts during the DACS imaging sets that could not be adequately corrected. The final data analysis therefore included 20 subjects (average age, $24.6 \pm 6.6 \mathrm{y} ; 7$ female and 13 male).

\section{Total Gastric Emptying}

Initial preferential retention of solids in the proximal stomach (fundic accommodation) was seen in all subjects. Over time, all subjects showed progressive transit of the radiolabeled solid meal from the proximal to the distal stomach (Fig. 4).

Total mean gastric retention is summarized in Table 1. Mean total gastric retention was $39.8 \% \pm 12.8 \%$ at $2 \mathrm{~h}$ and $5.8 \% \pm 6.0 \%$ at $4 \mathrm{~h}$. These clinically used 2 - and 4 -h values for measuring gastric emptying were compared between the men and women. For the 13 men, retention was $37.7 \% \pm$ $13.4 \%$ at $2 \mathrm{~h}$ and $4.0 \% \pm 4.2 \%$ at $4 \mathrm{~h}$, whereas for the 7 women, retention was $43.5 \% \pm 11.3 \%$ at $2 \mathrm{~h}(P=0.142$ vs. men) and $8.8 \% \pm 7.7 \%$ at $4 \mathrm{~h}(P=0.142)$. The mean halftime for gastric emptying in all patients was $1.62 \pm 0.33 \mathrm{~h}$ : $1.54 \pm 0.32 \mathrm{~h}$ for the men and $1.75 \mathrm{~h} \pm 0.28 \mathrm{~h}$ for the women.

\section{IMD Based on Division of Stomach into Proximal and Distal Halves}

The regional percentage of solid meal retention at 0,30 , $60,120,180$, and $240 \mathrm{~min}$ is shown in Figure 4A. The measure of fundic accommodation, $\mathrm{IMD}^{0}$, based on division of the stomach into halves averaged $0.75 \pm 0.15$ (Table 1 ). Over time, the IMD progressively decreased, representing movement of the radiolabeled meal from the proximal stomach into the distal stomach and subsequent gastric emptying.

\section{DACS}

Using DACS, the time of first onset of regular and sustained antral contractions was obtained. On average, sustained and periodic antral contractions started at a mean of 11.24 $\pm 12.98 \mathrm{~min}$ after meal ingestion. Figure 3 shows the typical conversion from irregular, nonperiodic to sustained, regular contractions.

Antral contraction frequency peaked during the DACS image set taken 30 min after meal ingestion, at $3.30 \pm 0.71 /$ min. This 30-min interval after meal ingestion was also the time when distal antral filling was the greatest $(36.65 \% \pm$ $13.49 \%$ based on proximal vs. distal division into halves and $25.98 \% \pm 15.10 \%$ based on Fourier separation) (Figs. 4A and 4B). The ejection fraction also peaked during the DACS image set taken 30 min after meal ingestion $(30.31 \% \pm$ $13.69 \%$ ejection fraction). 

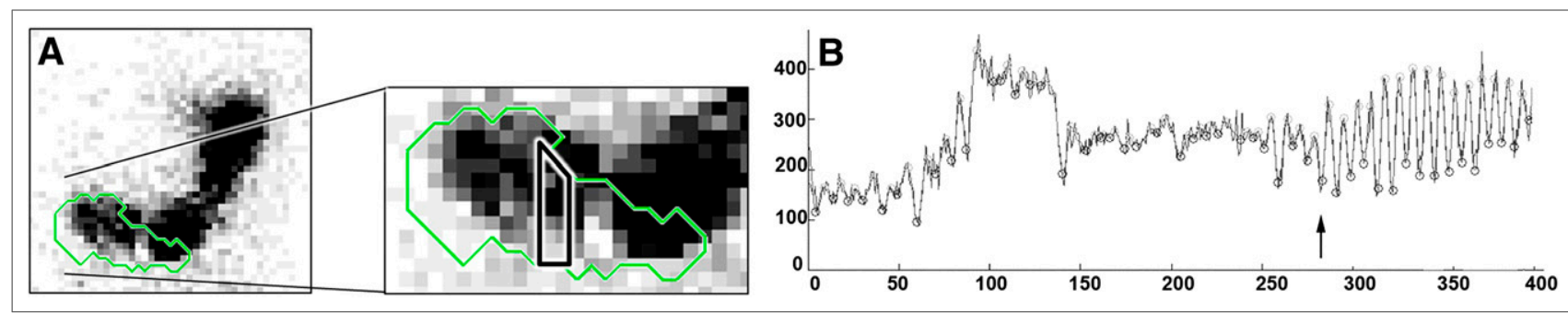

FIGURE 3. Time to onset of antral contractions. (A) Vertical rectangular ROI is placed over midportion of gastric antrum from one frame of serial anterior dynamic images used to generate time-activity curves shown in B. (B) $y$-axis is counts generated in antral $\mathrm{ROI}$ shown in A over time. $x$-axis is frame number, with 400 frames at $3 \mathrm{~s}$ per image. Time to onset of regular antral contractions is seen at image 278 or at $13.9 \mathrm{~min}$ (arrow) after immediately-after-meal static images.

The average speed of contractions from 0 to 80 min after meal ingestion was $3.16 \mathrm{~mm} / \mathrm{s}$. The mean location of the onset of antral contractions was in the midbody of the stomach, with the average location of onset of contractions being $40.54 \% \pm 10.78 \%$ from the distal to the proximal stomach along the longitudinal axis.

\section{IMD Based on DACS and Other Derived Measures of Antral Contraction}

$\mathrm{IMD}^{0}$ calculated by segmenting the proximal stomach from the antrum by defining the antrum by those pixels demonstrating phasic antral contractions was $0.85 \pm 0.14$, which was greater than the mean value for $\mathrm{IMD}^{0}$ based on gastric division into halves $(0.75 \pm 0.15)(P=0.004)$. There was a moderate correlation between $\mathrm{IMD}^{0}$ calculated by the two methods $(r=0.465 ; P<0.05)$.

There were no significant sex differences between the male and female values for DACS-derived $\operatorname{IMD}^{0}(P=0.13)$ - either for antral contraction frequency at $0 \min (P=0.34), 30 \mathrm{~min}$ $(P=0.48)$, or $60 \min (P=0.22)$ or for antral ejection fraction at $0 \min (P=0.13), 30 \mathrm{~min}(P=0.34)$, or $60 \mathrm{~min}(P=0.44)$.

\section{DISCUSSION}

The purpose of this study was to develop and obtain reference values for a GES study that goes beyond assessment of just total gastric emptying. Antral contractility was assessed in this study by adding continuous dynamic imaging or DACS after each static imaging set. IMD and DACS were analyzed using software developed in Matlab, which is readily available.

As in any first investigation of a new technique, we recognize that our results are limited to a small number of healthy subjects and will require validation and expansion to a larger number of healthy subjects. Because the healthy volunteers were all young (average age, $25 \mathrm{y}$ ), we are not able to look at age-related differences. There were no significant sex differences found for gastric retention or for the DACS-derived measurements of $\mathrm{IMD}^{0}$, antral contraction frequency, or ejection fraction.

We compared two measurements of $\mathrm{IMD}^{0}$, which assesses fundic accommodation. IMD is a reflection of regional gastric motility, with IMD immediately after meal ingestion ( $\mathrm{IMD}^{0}$ ) being used to assess fundic accommodation (6). In this study, $\mathrm{IMD}^{0}$ using the proximal half of the stomach averaged $0.75 \pm 0.15$. We previously established a lower limit of 0.568 for normal $\mathrm{IMD}^{0}$ when the stomach is halved using receiver operating curves to define sensitivity and specificity in a large group of patients. In that study, also on several healthy subjects, $\mathrm{IMD}^{0}$ averaged $0.672 \pm$ 0.092 (6). As shown in Table 1, the mean reference range for $\mathrm{IMD}^{0}$ using phasic contractions to define the antrum ranged from 0.71 to 0.99 .
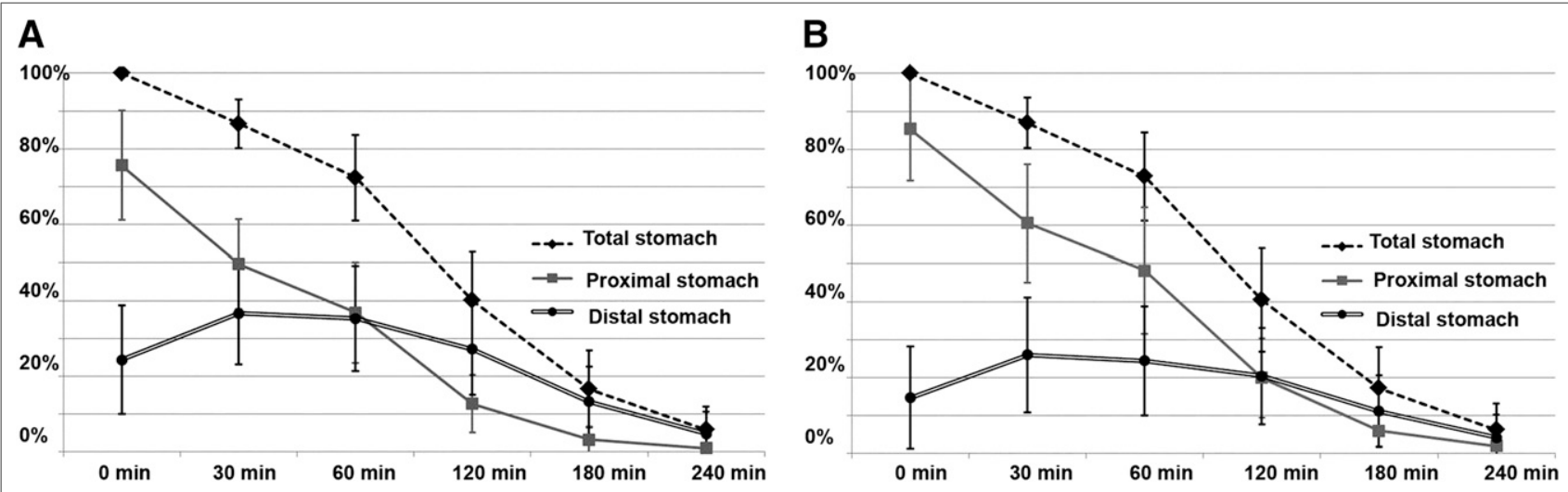

FIGURE 4. (A) Total and regional gastric retention over time using anatomic division of stomach into halves. (B) Total and regional gastric retention over time using Fourier frequency and amplitude to separate antrum from remaining proximal stomach. 
TABLE 1

Results Summary ( $n=20$ Healthy Subjects)

\begin{tabular}{|c|c|c|c|c|c|c|}
\hline Parameter & $0 \mathrm{~min}$ & $30 \mathrm{~min}$ & $60 \mathrm{~min}$ & $120 \mathrm{~min}$ & $180 \mathrm{~min}$ & $240 \min$ \\
\hline $\begin{array}{l}\text { IMD: gastric division into proximal } \\
\text { and distal halves }\end{array}$ & $0.76 \pm 0.14$ & $0.50 \pm 0.12$ & $0.37 \pm 0.13$ & $0.13 \pm 0.08$ & $0.03 \pm 0.04$ & $0.01 \pm 0.01$ \\
\hline $\begin{array}{l}\text { IMD: gastric division into proximal } \\
\text { and distal segments with Fourier-defined } \\
\text { antral region }\end{array}$ & $0.85 \pm 0.14$ & $0.61 \pm 0.16$ & $0.48 \pm 0.17$ & $0.20 \pm 0.10$ & $0.06 \pm 0.04$ & $0.02 \pm 0.02$ \\
\hline Total gastric retention (\%) & $100.0 \pm 0.0$ & $86.2 \pm 6.9$ & $72.1 \pm 11.5$ & $39.8 \pm 12.8$ & $16.4 \pm 9.9$ & $5.8 \pm 6.0$ \\
\hline Antral frequency (cycles/min) & $3.09 \pm 0.31$ & $3.30 \pm 0.73$ & $3.15 \pm 0.28$ & $2.96 \pm 0.35$ & NA & NA \\
\hline Antral ejection fraction (\%) & $26.28 \pm 13.74$ & $29.43 \pm 13.42$ & $27.96 \pm 15.31$ & $26.95 \pm 9.38$ & NA & NA \\
\hline Contraction speed $(\mathrm{mm} / \mathrm{s})$ & $2.91 \pm 1.89$ & $3.04 \pm 1.65$ & $3.54 \pm 0.90$ & $2.67 \pm 1.37$ & NA & NA \\
\hline \multicolumn{7}{|c|}{$\begin{array}{l}\mathrm{IMD}=\text { ratio of count in proximal stomach to total gastric count immediately after meal ingestion; NA = not available because of lov } \\
\text { ount caused by majority of meal's having left stomach. } \\
\text { Data are mean } \pm S D \text {. }\end{array}$} \\
\hline
\end{tabular}

In this study, we assessed a potentially more physiologic method to measure $\mathrm{IMD}^{0}$ assuming that the stomach can be divided functionally by its phasic contractions into proximal and distal segments using DACS. This assessment is more physiologic than simple anatomic division of the stomach (18). The mean normal value for $\mathrm{IMD}^{0}$ obtained on the basis of DACS-derived antral contractions (0.85 \pm $0.14)$ is higher than that using anatomic division of the stomach into halves $(0.75 \pm 0.15 ; P=0.004)$ because of a smaller physiologic area of DACS-defined antral contractions in the distal stomach. The result is a larger defined area of proximal stomach. There was a moderate correlation between $\mathrm{IMD}^{0}$ calculated by the two methods.

Anatomic division of the stomach into halves has appeal because of its simplicity and ease of automation. We have shown in patients that measuring $\mathrm{IMD}^{0}$ using the proximal half of the stomach correlated best with increased early satiety (6). Using Fourier analysis and DACS to measure fundic accommodation response adds complexity to GES and will require further study in a larger patient population to determine whether it correlates better with symptoms and other physiologic tests of fundic accommodation.

DACS provides additional measures of antral contractility. The use of fast Fourier transformation permits measurements of both the frequency and the amplitude of contractions. The frequency of antral contractions was highest on average (3.30 $\pm 0.73 / \mathrm{min})$ at $30 \mathrm{~min}$ after meal ingestion. The ejection fraction was also highest at $30 \mathrm{~min}, 29.43 \% \pm 13.42 \%$, suggesting that the most prominent contractions occur early, between 30 and $50 \mathrm{~min}$ after ingestion. The speed of contractions was highest $60 \mathrm{~min}$ after ingestion, and the average speed of antral contractions was $3.54 \pm 0.90 \mathrm{~mm} / \mathrm{s}$ at that time. Electrogastrography can also provide information on the frequency of gastric contractions (19). Although both DACS and electrogastrography can provide information on the frequency of antral contractions, electrogastrography does not provide information on the amplitude or strength of antral contractions or directly measure the speed of contraction.
The imaging protocol used in this study allows more complete characterization of proximal stomach fundic accommodation and distal stomach antral motility. Obtaining quantitative measures of fundic accommodation and antral contractility from GES images provides a quantitative and noninvasive method to provide more information on gastric motility and potential pathophysiology in symptomatic patients. Studies that assess fundic accommodation and antral contractility have already proven beneficial in explaining sex-related differences in gastric motility $(16,20)$.

We recognize that adding measurement of IMD and impaired fundic accommodation adds time and complexity to the acquisition and processing of GES data. To answer whether this added complexity is worthwhile, one needs to understand the clinical limitations of solid-meal GES as currently performed. It has been shown that $25 \%$ of patients with functional dyspepsia sent for GES studies have a normal conventional gastric emptying result that fails to direct the ordering physician to a treatable cause of the patient's symptoms (3). These patients are left without a diagnosis and potential for treatment. It is further estimated that approximately $40 \%$ of patients with functional dyspepsia have impaired fundic accommodation (11). Studies have shown that therapy with pharmacologic agents that relax the fundus can improve impaired fundic accommodation symptoms in these patients (2123). Therefore, accurate measurement of IMD to assess for abnormal fundic accommodation as a cause of a patient's symptoms can direct therapy for a significant number of patients. Other drugs have been shown to specifically increase antral contractility (24-26).

Several methods have been proposed for measuring IMD and impaired fundic accommodation as a part of a solidmeal GES study. These include simple visual assessment or quantification of the GES static images by division of the stomach into halves or thirds (6). It is unclear at this time whether a GES assessment of fundic accommodation with measurement of IMD should be done only after a normal GES result. A final determination of whether our proposed 
more physiologic method of defining the antrum by DACS and whether measurement of fundic accommodation is used before or after a normal GES study will require further study as a part of future larger clinical trials.

\section{CONCLUSION}

We have investigated a more comprehensive GES study combining both conventional static GES with DACS in healthy volunteers. This protocol provided assessment of IMD and antral contractility along with total gastric emptying and allows for a more physiologic division of the proximal versus the distal stomach. These reference results permit comparison to symptomatic patients to delineate potential gastric motility abnormalities causing patient symptoms. Broader clinical use will require further multicenter development of reference values and investigation of how this more comprehensive testing may improve diagnosis and aid targeted treatment of patients with upper gastrointestinal symptoms of dyspepsia and gastroparesis.

\section{DISCLOSURE}

No potential conflict of interest relevant to this article was reported.

\section{REFERENCES}

1. Abell TL, Camilleri M, Donohoe K, et al. Consensus recommendations for gastric emptying scintigraphy. Am J Gastroenterol. 2008;103:753-763.

2. Donohoe KJ, Maurer AH, Ziessman HA, et al. Procedure guideline for adult gastric emptying study 3.0. J Nucl Med Technol. 2009;37:196-200.

3. Pasricha PJ, Colvin R, Yates K, et al. Characteristics of patients with chronic unexplained nausea and vomiting and normal gastric emptying. Clin Gastroenterol Hepatol. 2011;9:567-576.

4. Abell TL, Camilleri M, Donohoe K, et al. Consensus recommendations for gastric emptying scintigraphy. Am J Gastroenterol. 2008;103:753-763.

5. Piessevaux H, Tack J, Walrand S, Pauwels S, Geubel A. Intragastric distribution of a standardized meal in health and functional dyspepsia: correlation with specific symptoms. Neurogastroenterol Motil. 2003;15:447-455.

6. Orthey $\mathrm{P}, \mathrm{Yu} \mathrm{D}$, Van Natta ML, et al. Intragastric meal distribution during gastric emptying scintigraphy for assessment of fundic accommodation: correlation with symptoms of gastroparesis. J Nucl Med. 2018;59:691-697.

7. Tomita T, Okugawa T, Yamasaki T, et al. Use of scintigraphy to evaluate gastric accommodation and emptying: comparison with barostat. J Gastroenterol Hepatol. 2013;28:106-111.
8. Urbain JL, Vekemans MC, Bouillon R, et al. Characterization of gastric antral motility disturbances in diabetes using a scintigraphic technique. J Nucl Med. 1993;34:576-581.

9. Parkman HP, Miller MA, Trate DM, et al. Effect of gastric acid suppressants on human gastric motility. Gut. 1998;42:243-250.

10. Parkman HP, Trate DM, Knight LC, Brown KL, Maurer AH, Fisher RS. Cholinergic effects on human gastric motility. Gut. 1999;45:346-354.

11. Kindt $S$, Tack J. Impaired gastric accommodation and its role in dyspepsia. Gut. 2006;55:1685-1691.

12. Troncon LE, Bennett RJ, Ahluwalia NK, Thompson DG. Abnormal intragastric distribution of food during gastric emptying in functional dyspepsia patients. Gut. 1994;35:327-332.

13. Tack J, Piessevaux H, Coulie B, et al. Role of impaired gastric accommodation to a meal in functional dyspepsia. Gastroenterology. 1998;115:1346-1352.

14. Ang D. Viewpoint: Measurement of gastric accommodation response: a reappraisal of conventional and emerging modalities. Neurogastroenterol Motil. 2011; 23:287-291.

15. Tougas G, Eaker EY, Abell TL, et al. Assessment of gastric emptying using a low fat meal: establishment of international control values. Am J Gastroenterol. 2000;95: 1456-1462.

16. Knight LC, Parkman HP, Miller MA, et al. Delayed gastric emptying in normal women is associated with decreased antral contractility. Am J Gastroenterol. 1997;92:968-975.

17. Altman DG. Practical Statistics for Medical Research. London, U.K.: Chapman and Hall; 1991.

18. Kelly KA. Gastric emptying of liquids and solids: roles of proximal and distal stomach. Am J Physiol. 1980;239:G71-G76.

19. Parkman HP, Hasler WL, Barnett JL, Eaker EY; American Motility Society Clinical GI Motility Testing Task Force. Electrogastrography: a document prepared by the gastric section of the American Motility Society Clinical GI Motility Testing Task Force. Neurogastroenterol Motil. 2003;15: 89-102.

20. Flier SN, Rose S. Is functional dyspepsia of particular concern in women? A review of gender differences in epidemiology, pathophysiologic mechanisms, clinical presentation, and management. Am J Gastroenterol. 2006; 101(12, suppl) S644-S653.

21. Tack J, Coulie B, Wilmer A, Andrioli A, Janssens J. Influence of sumatriptan on gastric fundus tone and on the perception of gastric distension in man. Gut. 2000;46:468-473.

22. Sekino Y, Vamada E, Sakai E, et al. Influence of sumatriptan on gastric accommodation and on antral contraction in healthy subjects assessed by ultrasonography. Neurogastroenterol Motil. 2012;24:1083-e564.

23. Tack J, Janssen P, Masaoka T, Farré R, Van Oudenhove L. Efficacy of buspirone, a fundus-relaxing drug, in patients with functional dyspepsia. Clin Gastroenterol Hepatol. 2012;10:1239-1245.

24. Coulie B, Tack J, Peeters T, Janssens J. Involvement of two different pathways in the motor effects of erythromycin on the gastric antrum in humans. Gut. 1998;43: $395-400$.

25. Tack J, Lee KJJ. Pathophysiology and treatment of functional dyspepsia. J Clin Gastroenterol. 2005; 39(5, suppl 3)S211-S216.

26. Chedid V, Camilleri M. Relamorelin for the treatment of gastrointestinal motility disorders. Expert Opin Investig Drugs. 2017;26:1189-1197. 